\title{
Consensus of Second-Order Multiagent Systems with Directed Signed Networks and Communication Delays
}

\author{
Huiqin Pei (iD) and Qiang Lai (iD \\ School of Electrical and Automation Engineering, East China Jiaotong University, Nanchang 330013, China \\ Correspondence should be addressed to Huiqin Pei; peihuiqinnx@126.com
}

Received 30 March 2020; Accepted 13 July 2020; Published 29 September 2020

Academic Editor: Guang Li

Copyright ( 92020 Huiqin Pei and Qiang Lai. This is an open access article distributed under the Creative Commons Attribution License, which permits unrestricted use, distribution, and reproduction in any medium, provided the original work is properly cited.

\begin{abstract}
This paper is concerned with the consensus problem for second-order multiagent systems (MASs) with directed signed networks and local communication delays. First, under a strongly connected directed graph, some properties for signed digraph and the model of second-order MASs are provided. A distributed nonlinear consensus control protocol is proposed for implementing consensus of second-order MASs with signed digraph and communication delay. Then, for structurally balanced and unbalanced networks, the consensus of second-order MASs with directed signed networks and local delays is proved via the Lyapunov method and Barbalat lemma. Simulation examples are given to demonstrate the effectiveness of our results.
\end{abstract}

\section{Introduction}

The cooperative control idea of MASs generally originates from gregarious behaviors of organisms in nature and society, such as flocking of migrating birds [1], swarming of foragers, and the synchronized behavior of a shoal of fish. These behaviors can help organisms to avoid predators and look for food, which contribute to the implementation of complex global behaviors. Consensus is known as an important fundamental branch of cooperative control of MASs, which means all agents can be assured to reach the same state value for any initial states of agents. Moreover, it has a wide range of applications, such as mobile robots [2], unmanned aerial vehicle [3], and sensor networks [4].

For consensus of MASs [5-10], many studies involve system graphs with all positive edge weights. When the MASs with signed graphs having positive and negative edge weights are taken into account, it is not easy to make all agents achieve the same state value. There are coordination and antagonistic contradiction between agents. In reality, there normally exist networks with signed graphs, e.g., the UAV network with bidirectional flying and the human social network. So the system studied can be seen as a signed network, and it is necessary to consider bipartite consensus of the system, which requests the agents to reach an agreement for agents in modulus but not in sign [11]. In [12], the bipartite consensus of MASs with first-order dynamics was investigated under undirected signed graphs and signed digraphs; e.g., all agents converge to an absolute value with two opposite fronts. Furthermore, the interval bipartite consensus of networked agents was studied, where the signed digraphs only need to include spanning trees in [13]. Subsequently, the finite time bipartite consensus issue of first-order MASs was researched under undirected signed graph in [14-17].

In practice, it is known that there are communication delays between agents, which affect the stability and performance of MASs. As we know, there are correlatively few research studies on the bipartite consensus of MASs with time delays. Hence, in this paper, the consensus problem of second-order MASs is investigated under directed signed networks and local communication delays. Compared with research contents in $[13,17]$, the second-order dynamical models of agents are considered. Meanwhile, a new distributed nonlinear consensus control protocol with local communication delays is put forward. The main contributions of the paper are as follows: (1) a distributed nonlinear consensus control protocol is put forward for implementing 
consensus of second-order MASs with signed digraph and communication delays; (2) for structurally balanced and unbalanced networks, the corresponding theorem is provided for the consensus of second-order MASs with directed signed networks and local delays about any initial conditions; (3) it is proved through the Lyapunov method and Barbalat lemma.

The reminder of the paper is organized as follows. Section 2 includes preliminaries of signed graph and problem statement. Section 3 includes main results of this paper, which contain the proposed distributed nonlinear control protocol to achieve the consensus of second-order MASs with directed signed networks and communication delays and the corresponding theorem and so on. Section 4 gives simulation examples to illustrate the validity of our results. Finally, Section 5 is the conclusion of this paper.

Notation 1. Some standard notations are listed as follows that are used in this paper. $R$ denotes real numbers. $R^{N}$ and $R^{N \times N}$ are the $N$-dimensional real vector space and $N \times N$ real matrix space, respectively. The $\operatorname{sign}(\sigma)$ shows the sign function with $\sigma \in R$, as follows:

$$
\operatorname{sign}(\sigma)= \begin{cases}1, & \sigma>0, \\ 0, & \sigma=0, \\ -1, & \sigma<0\end{cases}
$$

\section{Preliminaries and Problem Statement}

2.1. Preliminaries of the Signed Graph. A signed graph $G=$ $(V, E, A)$ includes a vertex set $V=\left\{v_{1}, v_{2}, \ldots, v_{N}\right\}$, an edge set $E \subseteq V \times V$, and a signed weighted adjacency matrix $A=\left(A_{i j}\right) \in R^{N \times N}$. Thereinto, an edge $E_{i j}=\left(v_{j}, v_{i}\right) \in E$ denotes the flow of information from $v_{j}$ to $v_{i}$. The corresponding signed weight $A_{i j}$ is positive or negative; that is, $A_{i j} \neq 0$, otherwise $A_{i j}=0$. Suppose that the graph $G$ has no self-loop, i.e., $A_{i i}=0, i \in 1,2, \ldots, N$. When $\left(v_{j}, v_{i}\right) \in E$, $\left(v_{i}, v_{j}\right) \in E$, and $A^{T}=A$, the graph $G$ is undirected. Conversely, it is called a directed graph or digraph. When $\bar{V} \subseteq V$ and $\bar{E} \subseteq E$, a directed graph $\bar{G}=(\bar{V}, t \bar{E} n, q \bar{A})$ is a subgraph of $G$. A path is a series of edges $\left(v_{k}, v_{k_{1}}\right),\left(v_{k_{1}}, v_{k_{2}}\right), \ldots,\left(v_{k_{n}}, v_{l}\right)$ from vertex $v_{l}$ to $v_{k}$ in $G$ with distinct vertices $v_{k_{i}}, i=1,2,3, \ldots, n$. A cycle is a special path with the starting and ending vertex at the same vertex. For a cycle, when the product of all signed edge weights is positive, the cycle is positive, otherwise negative.

Lemma 1 (see [18]). For a strongly connected directed graph $G$ with positive $A=\left(A_{i j}\right) \in R^{N \times N}$, the Laplacian matrix is represented as $L=\left(l_{i j}\right) \in R^{N \times N}$, where

$$
l_{i j}=\left\{-\sum_{l=1, l \neq i}^{N} A_{i l}, i=j, A_{i j}, i \neq j .\right.
$$

Then, there is $s>0$ to make $s^{T} L=0$.

Definition 1 (see [12]). When a signed graph $G$ allows a bipartition of the vertexes, that is, $V_{1}$ and $V_{2}$ with $V_{1} \cup V_{2}=$ $V$ and $V_{1} \cap V_{2}=0$, the signed graph $G$ is known as structurally balanced, i.e., $A_{i j}>0, v_{i}, v_{j} \in V_{1}$, and $A_{i j}<0, v_{i} \in V_{1}, v_{j} \in V_{2}$.

Definition 2. Let $\bar{D}$ define a diagonal matrix: $\bar{D}=\left\{\operatorname{diag}(\delta) \mid \delta=\left(\delta_{1}, \delta_{2}, \ldots, \delta_{i}, \ldots, \delta_{N}\right), \delta_{i} \in\{ \pm 1\}\right\}$.

Lemma 2 (see $[12,15,18]$ ). For a strongly connected signed digraph $G$, the conditions of structural balance are shown as follows:

(1) All directed cycles are positive in $G$

(2) Arbitrary matrix $D \in \bar{D}$, and there exists $D A D$ being nonnegative

(3) L has an eigenvalue 0 , and $\operatorname{rank}(L)=N-1$

Lemma 3 (see $[12,15,18]$ ). For a strongly connected signed digraph $G$, the conditions of structural unbalance are shown as follows:

(1) At least one directed cycle is negative in $G$

(2) There exists no matrix $D \in \bar{D}$, and there is $D A D$ being nonnegative

(3) L has an eigenvalue 0 , and $\operatorname{rank}(L)=N-1$

2.2. Problem Statement. Consider a multiagent system with second-order agents and local communication delays under the directed signed network. Each agent has the following dynamics:

$$
\left\{\begin{array}{l}
\dot{\vec{p}}_{i}(t)=\widehat{v}_{i}(t), \\
\dot{\vec{v}}_{i}(t)=\widehat{u}_{i}(t), \quad i=1,2, \ldots, N
\end{array}\right.
$$

where $\widehat{p}_{i}(t) \in R^{n}$ and $\widehat{v}_{i}(t) \in R^{n}$ represent the position state and velocity state of agent $i$, separately, and $\bar{u}_{i}(t) \in R^{n}$ is the control input of agent $i$. In order to facilitate the analysis, assume that all agents are in 1-D space, namely, $\widehat{p}_{i}, \widehat{v}_{i}, \widehat{u}_{i} \in R$. However, the results of the paper can be extended to multidimensional space.

Definition 3. The consensus of second-order multiagent system (3) is considered to be achieved for any initial state values $\widehat{p}_{i}(0)$ and $\widehat{v}_{i}(0)$ if there exist

$$
\left\{\begin{array}{l}
\lim _{t \longrightarrow \infty}|| \widehat{p}_{i}|-| \widehat{p}_{j}||=0, \text { and }\left|\widehat{p}_{i}\right|=\left|\widehat{p}_{j}\right| \\
\lim _{t \longrightarrow \infty}|| \widehat{v}_{i}|-| \widehat{v}_{j}||=0, \text { and }\left|\widehat{v}_{i}\right|=\left|\widehat{v}_{j}\right| \\
i, j=1,2, \ldots, N
\end{array}\right.
$$

The objective of this paper is to design a distributed nonlinear consensus control protocol which is used to find the consensus of second-order MASs with signed digraph and communication delays and to prove the corresponding consensus of second-order MASs for structurally balanced and unbalanced networks through the Lyapunov method and Barbalat lemma, that is, the stability analysis of secondorder MASs. 


\section{Main Results}

In this section, the consensus is investigated for the secondorder multiagent system with strongly connected directed signed network. The communication delays of information flow between agents are considered. Taking into account multiagent system (3), the following distributed nonlinear consensus control protocol is put forward:

$$
\begin{array}{r}
\widehat{u}_{i}(t)=-a\left(\sum_{j=1}^{N}\left|A_{i j}\right|\left(\widehat{p}_{i}(t)-\operatorname{sign}\left(A_{i j}\right) \widehat{p}_{j}\left(t-\tau_{i j}\right)\right)\right) \\
-b\left(\sum_{j=1}^{N}\left|A_{i j}\right|\left(\widehat{v}_{i}(t)-\operatorname{sign}\left(A_{i j}\right) \widehat{v}_{j}\left(t-\tau_{i j}\right)\right)\right), \\
i=1,2, \ldots, N,
\end{array}
$$

where $a$ and $b$ are nonnegative coupling coefficients, $\tau_{i j}$ denotes the communication delay from vertex $v_{j}$ to $v_{i}$ with $i \neq j$, and $\tau_{i i}=0$.
Theorem 1. Consider second-order multiagent system (3) with a strongly connected directed signed graph $G$ for any finite communication delay $\tau_{i j}$. For any initial conditions, when $G$ is structurally balanced, the bipartite consensus of the system can be asymptotically achieved. Similarly, when $G$ is structurally unbalanced, the consensus of the system can gradually come true.

Proof. For a signed digraph $G$ with strong connectivity, the diagonal matrix $D \in \bar{D}$ is chosen. Let $\xi(t)=D \widehat{p}(t)$ and $\eta(t)=D \widehat{v}(t)$; we have

$$
\begin{aligned}
& \xi_{i}(t)=\delta_{i} \widehat{p}_{i}(t), \\
& \eta_{i}(t)=\delta_{i} \widehat{v}_{i}(t),
\end{aligned}
$$

$$
i=1,2, \ldots, N \text {. }
$$

Through (6), expression (7) is obtained from system (3) and nonlinear control protocol (5):

$$
\left\{\begin{array}{l}
\dot{\xi}_{i}(t)=\eta_{i}(t), \quad i=1,2, \ldots, N \\
\delta_{i} \dot{\eta}_{i}(t)=-a\left(\sum_{j=1}^{N}\left|A_{i j}\right|\left(\delta_{i} \xi_{i}(t)-\operatorname{sign}\left(A_{i j}\right) \delta_{j} \xi_{j}\left(t-\tau_{i j}\right)\right)\right)-b\left(\sum_{j=1}^{N}\left|A_{i j}\right|\left(\delta_{i} \eta_{i}(t)-\operatorname{sign}\left(A_{i j}\right) \delta_{j} \eta_{j}\left(t-\tau_{i j}\right)\right)\right) .
\end{array}\right.
$$

When the directed signed network of the system is structurally balanced, according to Lemma 2, there exists $D A D$ with all nonnegative elements, and one has $\delta_{i} \operatorname{sign}\left(A_{i j}\right) \delta_{j}=\operatorname{sign}\left(\left|A_{i j}\right|\right)=1$. Then, via $\delta_{i} \operatorname{sign}\left(A_{i j}\right) \delta_{j}=1$ and $\delta_{i}^{2}=1$, expression (7) is rewritten as

$$
\left\{\begin{array}{l}
\dot{\xi}_{i}(t)=\eta_{i}(t) \\
\dot{\eta}_{i}(t)=-a\left(\sum_{j=1}^{N}\left|A_{i j}\right|\left(\xi_{i}(t)-\xi_{j}\left(t-\tau_{i j}\right)\right)\right)-b\left(\sum_{j=1}^{N}\left|A_{i j}\right|\left(\eta_{i}(t)-\eta_{j}\left(t-\tau_{i j}\right)\right)\right), \quad i=1,2, \ldots, N .
\end{array}\right.
$$

Let $A^{+}=\left(A_{i j}^{+}\right)_{N \times N}$, where $A_{i j}^{+}=\left|A_{i j}\right|$, for all $i, j=1,2, \ldots, N$. The corresponding signed digraph is marked as $G^{+}$with strongly connected and nonnegative weights. Then, by (8), there is

$$
\left\{\begin{array}{l}
\dot{\xi}_{i}(t)=\eta_{i}(t) \\
\dot{\eta}_{i}(t)=-a\left(\sum_{j=1}^{N} A_{i j}^{+}\left(\xi_{i}(t)-\xi_{j}\left(t-\tau_{i j}\right)\right)\right)-b\left(\sum_{j=1}^{N} A_{i j}^{+}\left(\eta_{i}(t)-\eta_{j}\left(t-\tau_{i j}\right)\right)\right), \quad i=1,2, \ldots, N .
\end{array}\right.
$$



that

According to Lemma 1, one has $s^{T} L=0$, which implies

$$
s_{i} l_{i i}=-\sum_{j=1, j \neq i}^{N} s_{j} l_{j i} .
$$

In addition, on account of $l_{i i}=-\sum_{j=1}^{N} A_{i j}^{+}$, it can be acquired that

$$
\begin{aligned}
& \sum_{j=1}^{N} s_{i} A_{i j}^{+}=\sum_{j=1}^{N} s_{j} A_{j i}^{+}, \\
& \sum_{i=1}^{N} s_{j} A_{j i}^{+}=\sum_{i=1}^{N} s_{i} A_{i j}^{+} .
\end{aligned}
$$

Take into account the following Lyapunov function:

$$
V(t)=V_{1}(t)+V_{2}(t)
$$

where

$$
\begin{aligned}
V_{1}(t) & =\frac{1}{2} \sum_{i=1}^{N} s_{i} \eta_{i}^{T}(t) \eta_{i}(t) \\
V_{2}(t) & =\frac{b}{2} \sum_{i=1}^{N} \sum_{j=1}^{N} \int_{t-\tau_{j i}}^{t} s_{j} A_{j i}^{+} \eta_{i}^{T}(\theta) \eta_{i}(\theta) \mathrm{d} \theta .
\end{aligned}
$$

Take the derivative of $V_{1}(t)$ and $V_{2}(t)$, and there exist

$$
\begin{aligned}
\dot{V}_{1}(t)= & \sum_{i=1}^{N} s_{i} \eta_{i}^{T}(t) \dot{\eta}_{i}(t) \\
= & \sum_{i=1}^{N} s_{i} \eta_{i}^{T}(t)\left[-a \sum_{j=1}^{N} A_{i j}^{+}\left(\xi_{i}(t)-\xi_{j}\left(t-\tau_{i j}\right)\right)\right. \\
& \left.-b \sum_{j=1}^{N} A_{i j}^{+}\left(\eta_{i}(t)-\eta_{j}\left(t-\tau_{i j}\right)\right)\right]
\end{aligned}
$$$$
\dot{V}_{2}(t)=\frac{b}{2} \sum_{i=1}^{N} \sum_{j=1}^{N} s_{j} A_{j i}^{+}\left[\eta_{i}^{T}(t) \eta_{i}(t)-\eta_{i}^{T}\left(t-\tau_{j i}\right) \eta_{i}\left(t-\tau_{j i}\right)\right]
$$$$
=\frac{b}{2} \sum_{i=1}^{N} \sum_{j=1}^{N} s_{i} A_{i j}^{+} \eta_{i}^{T}(t) \eta_{i}(t)-\frac{b}{2} \sum_{i=1}^{N} \sum_{j=1}^{N} s_{i} A_{i j}^{+} \eta_{j}^{T}\left(t-\tau_{i j}\right) \eta_{j}
$$$$
\cdot\left(t-\tau_{i j}\right) \text {. }
$$

Hence, by (15) and (16), we have
$\dot{V}(t)=\dot{V}_{1}(t)+\dot{V}_{2}(t)$

$$
\begin{aligned}
= & -a \sum_{i=1}^{N} \sum_{j=1}^{N} s_{i} A_{i j}^{+} \eta_{i}^{T}(t)\left[\xi_{i}(t)-\xi_{j}\left(t-\tau_{i j}\right)\right] \\
& -\frac{b}{2} \sum_{i=1}^{N} \sum_{j=1}^{N} s_{i} A_{i j}^{+}\left[\eta_{i}^{T}(t) \eta_{i}(t)-2 \eta_{i}^{T}(t) \eta_{j}\left(t-\tau_{i j}\right)\right. \\
& \left.+\eta_{j}^{T}\left(t-\tau_{i j}\right) \eta_{j}\left(t-\tau_{i j}\right)\right] \\
= & -a \sum_{i=1}^{N} \sum_{j=1}^{N} s_{j} A_{j i}^{+} \eta_{i}^{T}(t)\left[\xi_{i}(t)-\xi_{i}\left(t-\tau_{j i}\right)\right] \\
& -\frac{b}{2} \sum_{i=1}^{N} \sum_{j=1}^{N} s_{i} A_{i j}^{+}\left[\eta_{i}(t)-\eta_{j}\left(t-\tau_{i j}\right)\right]^{T} \\
& \cdot\left[\eta_{i}(t)-\eta_{j}\left(t-\tau_{i j}\right)\right] \\
\leq & -a \sum_{i=1}^{N} \sum_{j=1}^{N} s_{j} A_{j i}^{+}\left[\xi_{i}(t)-\xi_{i}\left(t-\tau_{j i}\right)\right]^{T} \\
\tau_{j i} & \left.b \xi_{i}(t)-\xi_{i}\left(t-\tau_{j i}\right)\right] \\
& -\frac{b}{2} \sum_{i=1}^{N} \sum_{j=1}^{N} s_{j} A_{j i}^{+}\left[\eta_{i}(t)-\eta_{i}\left(t-\tau_{j i}\right)\right]^{T}\left[\eta_{i}(t)-\eta_{i}\left(t-\tau_{j i}\right)\right]
\end{aligned}
$$

$\leq 0$.

Therefore, $V(t)$ is descending and $V(t) \geq 0$ shows that $\lim _{t \rightarrow \infty} V(t)$ is finite. Furthermore, through (12), it is known that $\xi_{i}(t)$ and $\eta_{i}(t)$ are bounded for $i \in\{1,2, \ldots, N\}$. By system (9), for arbitrary $i \in\{1,2, \ldots, N\}$, we see that $\dot{\xi}_{i}(t)$ and $\dot{\eta}_{i}(t)$ are bounded. So, it is concludedd that $\ddot{V}(t)$ is also bounded. According to Barbalat lemma [19], it is obtained that $\lim _{t \rightarrow \infty}$ as $_{j} A_{j i}^{+}\left(\left[\xi_{i}(t)-\xi_{i}\left(t-\tau_{j i}\right)\right]^{T} / \tau_{j i}\right)\left[\xi_{i}(t)-\right.$ $\left.\xi_{i}\left(t-\tau_{j i}\right)\right]=0 \quad$ and $\quad \lim _{t \rightarrow \infty}(b / 2) s_{j} A_{j i}^{+}\left[\eta_{i}(t)-\eta_{i}\right.$ $\left.\left(t-\tau_{j i}\right)\right]^{T}\left[\eta_{i}(t)-\eta_{i}\left(t-\tau_{j i}\right)\right]=0$. On account of $a>0, b>0, s>0$, and $A_{j i}^{+}>0$,

$$
\begin{aligned}
& \lim _{t \longrightarrow \infty}\left[\xi_{i}(t)-\xi_{i}\left(t-\tau_{j i}\right)\right]=0, \\
& \lim _{t \longrightarrow \infty}\left[\eta_{i}(t)-\eta_{i}\left(t-\tau_{j i}\right)\right]=0 .
\end{aligned}
$$

Moreover, there are $\dot{\xi}_{i}(t) \longrightarrow 0$ and $\dot{\eta}_{i}(t) \longrightarrow 0$ for $i \in\{1,2, \ldots, N\}$.

Because the signed network is strongly connected, it is observed that $\dot{\xi}_{i}(t) \longrightarrow 0$ and $\dot{\eta}_{i}(t) \longrightarrow 0$ when $t \longrightarrow \infty$ for $i \in\{1,2, \ldots, N\}$. So, $\xi_{i}(t)$ and $\eta_{i}(t)$ tend to be steady values $c_{i}, d_{i} \in R$, respectively. Since the matrix $A^{+}$is irreducible, the 
largest invariant sets of system (9) are $\Phi_{1}=\left\{\xi_{1}(t)\right.$, $\left.\xi_{2}(t), \ldots, \xi_{N}(t) \mid \quad \xi_{1}(t)=\xi_{2}(t)=\cdots=\xi_{N}(t)\right\}$ and $\Phi_{2}=$ $\left\{\eta_{1}(t), \eta_{2}(t), \ldots, \eta_{N}(t) \mid \eta_{1}(t)=\eta_{2}(t)=\cdots=\eta_{N}(t)\right\}$. There exist constants $c \in R$ and $d \in R$, such that $\xi_{i}(t) \longrightarrow c$ and $\eta_{i}(t) \longrightarrow d$ as $t \longrightarrow \infty$ for $i \in\{1,2, \ldots, N\}$. Therefore, for system (9) with any finite communication delay $\tau_{i j}$ and initial values, we have $\lim _{t \longrightarrow \infty} \xi_{i}(t) \longrightarrow c$ and $\lim _{t \longrightarrow \infty} \eta_{i}(t) \longrightarrow d=0$. As a result, the position $\widehat{p}_{i}(t)$ and velocity $\widehat{v}_{i}(t)$ states of system (3) are satisfied with expression (4) for $i=\{1,2, \ldots N\}$. Then, the bipartite consensus of system (3) can be asymptotically realized for the signed network with structural balance.

When the directed signed network of the system is structurally unbalanced, the corresponding graph $G$ includes one or more negative cycles from Lemma 3. In order to facilitate the analysis, assume that $G$ only include one negative cycle with an negative weight edge $\left(v_{j 0}, v_{i 0}\right)$, $A_{i 0, j 0}<0$. Thus, the subgraph $\bar{G}$ of $G$ is structurally balanced to exclude the edge $\left(v_{j_{0}}, v_{i_{0}}\right)$. From Definition 1 , the signed digraph $\bar{G}$ has a bipartition of the vertexes $\bar{V}_{1}$ and $\bar{V}_{2}$. Meanwhile, $v_{j_{0}}$ and $v_{i_{0}}$ belong to $\bar{V}_{1}$ or $\bar{V}_{2}$. Note that $\bar{V}_{1}=V_{1}$ and $\bar{V}_{2}=V_{2}$. At present, adding the edge $\left(v_{j_{0}}, v_{i_{0}}\right)$ to the subgraph $\bar{G}$, the bipartition of the vertexes $V_{1}$ and $V_{2}$ for $G$ is obtained. So, it is known that $D=\operatorname{diag}(\delta)$ with $\delta_{i}=$ 1 as $v_{i} \in V_{1}$ and $\delta_{i}=-1$ as $v_{i} \in V_{2}$. Then, one has DAD = $\left(A_{i j}^{0}\right)_{N \times N}$ with one negative element $\left(A_{i_{0} j_{0}}^{0}=\delta_{i_{0}} A_{i_{0} j_{0}} \delta_{j_{0}}=\right.$ $\left.A_{i_{0} j_{0}}<0\right)$.

Then, from (7), there exists

$$
\left\{\begin{array}{l}
\dot{\xi}_{i}(t)=\eta_{i}(t), \quad i=1,2, \ldots, N \\
\dot{\eta}_{i}(t)=-a\left(\sum_{j=1}^{N}\left|A_{i j}\right|\left(\xi_{i}(t)-\delta_{i} \operatorname{sign}\left(A_{i j}\right) \delta_{j} \xi_{j}\left(t-\tau_{i j}\right)\right)\right)-b\left(\sum_{j=1}^{N}\left|A_{i j}\right|\left(\eta_{i}(t)-\delta_{i} \operatorname{sign}\left(A_{i j}\right) \delta_{j} \eta_{j}\left(t-\tau_{i j}\right)\right)\right) .
\end{array}\right.
$$

Similarly, consider the Lyapunov function $V(t)=V_{1}(t)+V_{2}(t)$, where

$$
\begin{aligned}
& V_{1}(t)=\frac{1}{2} \sum_{i=1}^{N} s_{i} \eta_{i}^{T}(t) \eta_{i}(t), \\
& V_{2}(t)=\frac{b}{2} \sum_{i=1}^{N} \sum_{j=1}^{N} \int_{t-\tau_{j i}}^{t} s_{j}\left|A_{j i}\right| \eta_{i}^{T}(\theta) \eta_{i}(\theta) \mathrm{d} \theta .
\end{aligned}
$$

Here, define a nonnegative matrix $B^{+}=\left(B_{i j}^{+}\right)_{N \times N}$, where

$$
B_{i j}^{+}= \begin{cases}0, & i=i_{0}, j=j_{0}, \\ A_{i j}^{+}, & \text {else. }\end{cases}
$$

Take the derivative of $V_{1}(t)$ and $V_{2}(t)$, and it is obtained that

$$
\begin{aligned}
\dot{V}_{1}(t)= & \sum_{i=1}^{N} s_{i} \eta_{i}^{T}(t) \dot{\eta}_{i}(t) \\
= & \sum_{i=1}^{N} s_{i} \eta_{i}^{T}(t)\left(-a\left(\sum_{j=1}^{N}\left|A_{i j}\right|\left(\xi_{i}(t)-\delta_{i} \operatorname{sign}\left(A_{i j}^{+}\right) \delta_{j} \xi_{j}\left(t-\tau_{i j}\right)\right)\right)\right) \\
& +\sum_{i=1}^{N} s_{i} \eta_{i}^{T}(t)\left(-b\left(\sum_{j=1}^{N}\left|A_{i j}\right|\left(\eta_{i}(t)-\delta_{i} \operatorname{sign}\left(A_{i j}^{+}\right) \delta_{j} \eta_{j}\left(t-\tau_{i j}\right)\right)\right)\right) \\
& +s_{i_{0}} \eta_{i_{0}}^{T}(t) A_{i_{0} j_{0}}\left[a\left(\xi_{j_{0}}\left(t-\tau_{i_{0} j_{0}}\right)+\xi_{i_{0}}(t)\right)+b\left(\eta_{j_{0}}\left(t-\tau_{i_{0} j_{0}}\right)+\eta_{i_{0}}(t)\right)\right] \\
= & \sum_{i=1}^{N} \sum_{j=1}^{N} a s_{i} \eta_{i}^{T}(t) B_{i j}^{+}\left[\xi_{j}\left(t-\tau_{i j}\right)-\xi_{i}(t)\right]+\sum_{i=1}^{N} \sum_{j=1}^{N} b s_{i} \eta_{i}^{T}(t) B_{i j}^{+}\left[\eta_{j}\left(t-\tau_{i j}\right)-\eta_{i}(t)\right] \\
& +a s_{i_{0}} \eta_{i_{0}}^{T}(t) A_{i_{0} j_{0}}\left[\xi_{j_{0}}\left(t-\tau_{i_{0} j_{0}}\right)+\xi_{i_{0}}(t)\right]+b s_{i_{0}} \eta_{i_{0}}^{T}(t) A_{i_{0} j_{0}}\left[\eta_{j_{0}}\left(t-\tau_{i_{0} j_{0}}\right)+\eta_{i_{0}}(t)\right],
\end{aligned}
$$




$$
\begin{aligned}
\dot{V}_{2}(t)= & \frac{b}{2} \sum_{i=1}^{N} \sum_{j=1}^{N} s_{j}\left|A_{j i}\right|\left[\eta_{i}^{T}(t) \eta_{i}(t)-\eta_{i}^{T}\left(t-\tau_{j i}\right) \eta_{i}\left(t-\tau_{j i}\right)\right] \\
= & \frac{b}{2} \sum_{i=1}^{N} \sum_{j=1}^{N} s_{i} A_{i j}^{+} \eta_{i}^{T}(t) \eta_{i}(t)-\frac{b}{2} s_{i_{0}} A_{i_{0} j_{0}} \eta_{i_{0}}^{T}(t) \eta_{i_{0}}(t)-\frac{b}{2} \sum_{i=1}^{N} \sum_{j=1}^{N} s_{i} A_{i j}^{+} \eta_{j}^{T}\left(t-\tau_{i j}\right) \eta_{j}\left(t-\tau_{i j}\right) \\
& +\frac{b}{2} s_{i_{0}} A_{i_{0} j_{0}} \eta_{j_{0}}^{T}\left(t-\tau_{i_{0} j_{0}}\right) \eta_{j_{0}}\left(t-\tau_{i_{0} j_{0}}\right) \\
= & \frac{b}{2} \sum_{i=1}^{N} \sum_{j=1}^{N} s_{i} B_{i j}^{+} \eta_{i}^{T}(t) \eta_{i}(t)-\frac{b}{2} s_{i_{0}} A_{i_{0} j_{0}} \eta_{i_{0}}^{T}(t) \eta_{i_{0}}(t)-\frac{b}{2} \sum_{i=1}^{N} \sum_{j=1}^{N} s_{i} B_{i j}^{+} \eta_{j}^{T}\left(t-\tau_{i j}\right) \eta_{j}\left(t-\tau_{i j}\right) \\
& +\frac{b}{2} s_{i_{0}} A_{i_{0} j_{0}} \eta_{j_{0}}^{T}\left(t-\tau_{i_{0} j_{0}}\right) \eta_{j_{0}}\left(t-\tau_{i_{0} j_{0}}\right) .
\end{aligned}
$$

Thus, via (22) and (23), we have

$$
\begin{aligned}
\dot{V}(t)= & \dot{V}_{1}(t)+\dot{V}_{2}(t) \\
= & a \sum_{i=1}^{N} \sum_{j=1}^{N} s_{i} \eta_{i}^{T}(t) B_{i j}^{+}\left[\xi_{j}\left(t-\tau_{i j}\right)-\xi_{i}(t)\right]+a s_{i_{0}} A_{i_{0} j_{0}} \eta_{i_{0}}^{T}(t)\left[\xi_{j_{0}}\left(t-\tau_{i_{0} j_{0}}\right)+\xi_{i_{0}}(t)\right] \\
& -\frac{b}{2} \sum_{i=1}^{N} \sum_{j=1}^{N} s_{i} B_{i j}^{+}\left[\eta_{i}^{T}(t) \eta_{i}(t)-2 \eta_{i}^{T}(t) \eta_{j}\left(t-\tau_{i j}\right)+\eta_{j}^{T}\left(t-\tau_{i j}\right) \eta_{j}\left(t-\tau_{i j}\right)\right] \\
& +\frac{b}{2} s_{i_{0}} A_{i_{0} j_{0}}\left[\eta_{i_{0}}^{T}(t) \eta_{i_{0}}(t)+2 \eta_{i_{0}}^{T}(t) \eta_{j_{0}}\left(t-\tau_{i_{0} j_{0}}\right)+\eta_{j_{0}}^{T}\left(t-\tau_{i_{0} j_{0}}\right) \eta_{j_{0}}\left(t-\tau_{i_{0} j_{0}}\right)\right] \\
= & -a \sum_{i=1}^{N} \sum_{j=1}^{N} s_{j} B_{j i}^{+} \eta_{i}^{T}(t)\left[\xi_{i}(t)-\xi_{i}\left(t-\tau_{j i}\right)\right]+a s_{i_{0}} A_{i_{0} j_{0}} \eta_{i_{0}}^{T}(t)\left[\xi_{i_{0}}(t)+\xi_{j_{0}}\left(t-\tau_{i_{0} j_{0}}\right)\right] \\
& -\frac{b}{2} \sum_{i=1}^{N} \sum_{j=1}^{N} s_{i} B_{i j}^{+}\left[\eta_{i}(t)-\eta_{j}\left(t-\tau_{i j}\right)\right]^{T}\left[\eta_{i}(t)-\eta_{j}\left(t-\tau_{i j}\right)\right] \\
& +\frac{b}{2} s_{i_{0}} A_{i_{0} j_{0}}\left[\eta_{i_{0}}(t)+\eta_{j_{0}}\left(t-\tau_{i_{0} j_{0}}\right)\right]^{T}\left[\eta_{i_{0}}(t)+\eta_{j_{0}}\left(t-\tau_{i_{0} j_{0}}\right)\right] \\
\leq & -a \sum_{i=1}^{N} \sum_{j=1}^{N} s_{j} B_{j i}^{+}\left[\xi_{i}(t)-\xi_{i}\left(t-\tau_{j i}\right)\right]^{T}\left[\xi_{i}(t)-\xi_{i}\left(t-\tau_{j i}\right)\right]+a s_{i_{0}} A_{i_{0} j_{0}} \eta_{i_{0}}^{T}(t)\left[\xi_{i_{0}}(t)+\xi_{j_{0}}\left(t-\tau_{i_{0} j_{0}}\right)\right] \\
& -\frac{b}{2} \sum_{i=1}^{N} \sum_{j=1}^{N} s_{j} B_{j i}^{+}\left[\eta_{i}(t)-\eta_{i}\left(t-\tau_{j i}\right)\right]^{T}\left[\eta_{i}(t)-\eta_{i}\left(t-\tau_{j i}\right)\right] \\
& +\frac{b}{2} s_{i_{0}} A_{i_{0} j_{0}}\left[\eta_{i_{0}}(t)+\eta_{j_{0}}\left(t-\tau_{i_{0} j_{0}}\right)\right]^{T}\left[\eta_{i_{0}}(t)+\eta_{j_{0}}\left(t-\tau_{i_{0} j_{0}}\right)\right] \leq 0 . \\
&
\end{aligned}
$$

Hence, $V(t)$ is nonincreasing, and $V(t) \geq 0$ indicates that $\lim _{t \rightarrow \infty} V(t)$ is finite. Ulteriorly, via $V(t)$, it is known that $\xi_{i}(t)$ and $\eta_{i}(t)$ are bounded for $i \in\{1,2, \ldots, N\}$. Through system (19), for $\forall i \in\{1,2, \ldots, N\}, \quad \dot{\xi}_{i}(t)$ and $\dot{\eta}_{i}(t)$ are bounded. It is concluded that $\ddot{V}(t)$ is likewise bounded. Via Barbalat lemma [19], it is obvious that $\lim _{t \rightarrow \infty}$ $s_{i_{0}} A_{i_{0} j_{0}}\left[\eta_{i_{0}}(t)+\eta_{j_{0}}\left(t-\tau_{i_{0} j_{0}}\right)\right]^{T}\left[\eta_{i_{0}}(t)+\eta_{j_{0}}\left(t-\tau_{i_{0} j_{0}}\right)\right]=0$, and

$$
\begin{array}{r}
\lim _{t \longrightarrow \infty} a s_{i_{0}} A_{i_{0} j_{0}}\left[\xi_{i_{0}}(t)+\xi_{j_{0}}\left(t-\tau_{i_{0} j_{0}}\right)\right]=0, \\
\lim _{t \longrightarrow \infty} a s_{j} B_{j i}^{+} \frac{\left[\xi_{i}(t)-\xi_{i}\left(t-\tau_{j i}\right)\right]^{T}}{\tau_{j i}}\left[\xi_{i}(t)-\xi_{i}\left(t-\tau_{j i}\right)\right]=0, \\
\lim _{t \longrightarrow \infty} \frac{b}{2} s_{j} B_{j i}^{+}\left[\eta_{i}(t)-\eta_{i}\left(t-\tau_{j i}\right)\right]^{T}\left[\eta_{i}(t)-\eta_{i}\left(t-\tau_{j i}\right)\right]=0 .
\end{array}
$$


Because of $a, b>0, s_{i}, s_{i_{0}}>0, B_{j i}^{+}>0$, and $A_{i_{0} j_{0}}<0$, there $\quad$ Moreover, for system (19), one has are

$$
\begin{aligned}
\lim _{t \longrightarrow \infty}\left[\xi_{i}(t)-\xi_{i}\left(t-\tau_{j i}\right)\right] & =0, \\
\lim _{t \longrightarrow \infty}\left[\eta_{i}(t)-\eta_{i}\left(t-\tau_{j i}\right)\right] & =0, \\
\lim _{t \longrightarrow \infty}\left[\xi_{i_{0}}(t)+\xi_{j_{0}}\left(t-\tau_{i_{0} j_{0}}\right)\right] & =0, \\
\lim _{t \longrightarrow \infty}\left[\eta_{i_{0}}(t)+\eta_{j_{0}}\left(t-\tau_{i_{0} j_{0}}\right)\right] & =0 .
\end{aligned}
$$

$$
\begin{aligned}
& \left\{\begin{array}{l}
\dot{\xi}_{i_{0}}(t)=\eta_{i_{0}}(t), \\
\dot{\eta}_{i_{0}}(t)=-a\left(\sum_{j=1}^{N}\left|A_{i_{0} j}\right|\left(\xi_{i_{0}}(t)-\delta_{i_{0}} \operatorname{sign}\left(A_{i_{0} j}\right) \delta_{j} \xi_{j}\left(t-\tau_{i_{0} j}\right)\right)\right)-b\left(\sum_{j=1}^{N}\left|A_{i_{0} j}\right|\left(\eta_{i_{0}}(t)-\delta_{i_{0}} \operatorname{sign}\left(A_{i_{0} j}\right) \delta_{j}\right)\right. \\
\quad=a A_{i_{0} j_{0}}\left(\xi_{i_{0}}(t)+\xi_{j}\left(t-\tau_{i_{0} j}\right)\right)+b A_{i_{0} j_{0}}\left(\eta_{i_{0}}(t)+\eta_{j}\left(t-\tau_{i_{0} j}\right)\right)-a\left(\sum _ { j \neq j _ { 0 } } B _ { i _ { 0 } j } \left(\xi_{i_{0}}(t)-\xi_{j}(t\right.\right.
\end{array}\right. \\
& \left.\quad-b\left(\sum_{j \neq j_{0}} B_{i_{0} j}\left(\eta_{i_{0}}(t)-\eta_{j}\left(t-\tau_{i_{0} j}\right)\right)\right)\right) \\
& \left\{\begin{array}{l}
\dot{\xi}_{i}(t)=\eta_{i}(t), \quad i \in\{1,2, \ldots, N\}, \\
\dot{\eta}_{i}(t)=-a\left(\sum_{j=1}^{N} B_{i j}^{+}\left(\xi_{i}(t)-\xi_{j}\left(t-\tau_{i j}\right)\right)\right)-b\left(\sum_{j=1}^{N} B_{i j}^{+}\left(\eta_{i}(t)-\eta_{j}\left(t-\tau_{i j}\right)\right)\right) \longrightarrow 0 \text { ast } \longrightarrow \infty .
\end{array}\right.
\end{aligned}
$$

Then, there are $\dot{\xi}_{i}(t) \longrightarrow 0$ and $\dot{\eta}_{i}(t) \longrightarrow 0$ for $i \in\{1,2, \ldots, N\}$.

Here, $G\left(A^{+}\right)$is a strongly connected digraph, according to Lemma 4 in [16], and it is obtained that $G\left(B^{+}\right)$is a weakly connected digraph. Hence, one has $\xi_{1}(t)=$ $\xi_{2}(t)=\cdots=\xi_{N}(t)$ and $\eta_{1}(t)=\eta_{2}(t)=\cdots=\eta_{N}(t)$ as $t \longrightarrow \infty$. Meanwhile, there are $\xi_{i_{0}}(t)=$ $\xi_{j_{0}}(t)=0$ and $\eta_{i_{0}}(t)=\eta_{j_{0}}(t)=0$ as $t \longrightarrow \infty$. Finally, $p_{i}(t)=\delta_{i} \xi_{i}(t)=0$ and $v_{i}(t)=\delta_{i} \eta_{i}(t)=0$, as $t \longrightarrow \infty$ for $i=\{1,2, \ldots N\}$, which are satisfied with expression (4). So, under structural unbalance, the consensus of system (3) can gradually come true. When the signed digraph $G$ includes more cycles with negative weight, the above analysis approach is applicable.

Remark 1. In this paper, the finite communication delay, $\tau_{i j}$ or $\tau_{j i}$, is minor. In most cases, the excessive delay causes system instability. However, in some cases, the delay may have a stabilizing effect. In [20], Yu et al. researched consensus and quasi-consensus of multiagent systems. In the delayed position information case, the corresponding consensus can be achieved. In [21,22], Liu et al. proposed Bessel-Laguerre integral inequality, which was applied to stability analysis of linear systems with infinite distributed delays, and gave a survey on time-delay approach to networked control.

\section{Simulation}

In this section, simulation examples are given to demonstrate the effectiveness of our results.

Example 1. Under structural balance, a second-order strongly connected multiagent system (3) with signed digraph is taken into account as shown in Figure 1(a), which includes 5 agents. The corresponding adjacency matrix is represented as

$$
A_{a}=\left(\begin{array}{ccccc}
0 & 0 & 0 & 0 & 1.2 \\
1.5 & 0 & 0 & 0 & 0 \\
0 & -1 & 0 & 0 & 0 \\
0 & 0 & 1 & 0 & 0 \\
0 & 3 & 0 & -1.3 & 0
\end{array}\right) .
$$

Here, proposed nonlinear control protocol (5) with communication delays is used to find the consensus of system (3). Choose that all communication delays are $\tau_{15}=$ $0.1, \tau_{21}=0.3, \tau_{32}=0.25, \tau_{43}=0.15, \tau_{52}=0.1$, and $\tau_{54}=0.1$ 


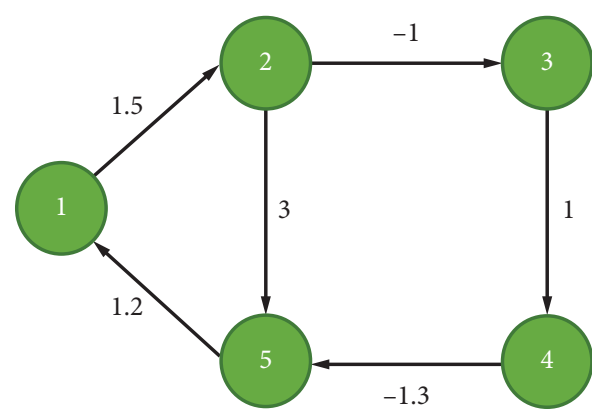

(a)

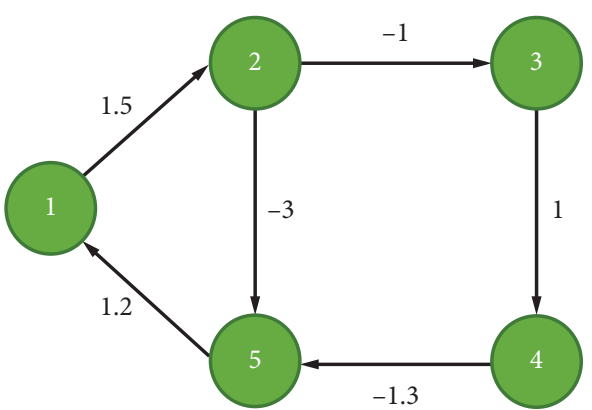

(b)

FIGURE 1: Strongly connected directed signed networks.

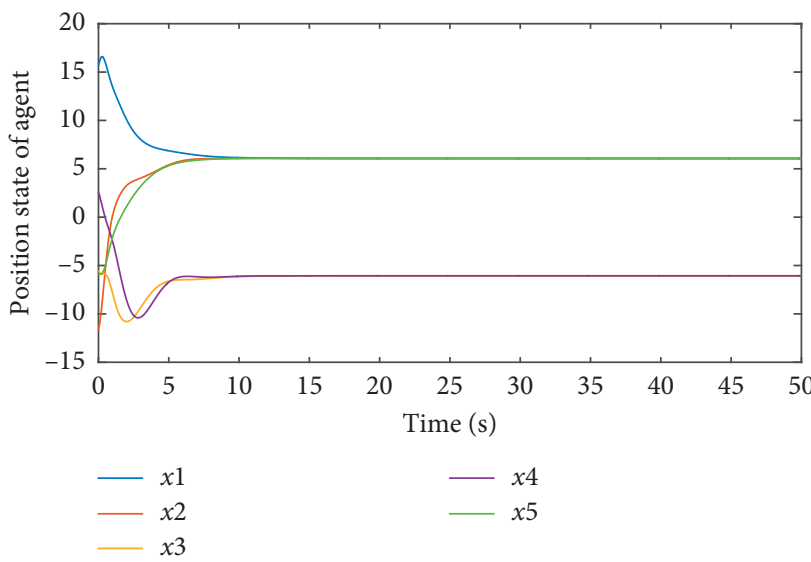

(a)

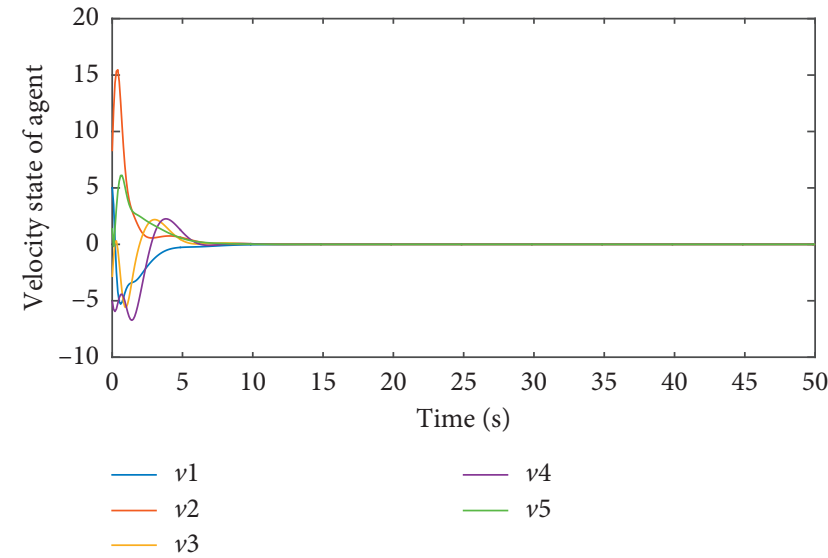

(b)

FIgURE 2: Position and velocity states of agents on the structurally balanced network in Figure 1(a).

and the nonnegative coupling coefficients are $a=1$ and $b=2$. The initial position and velocity states are $\widehat{p}(0)=(15,-12,-5,3,-7)^{T}$ and $\widehat{v}(0)=(7,3,-5,-4,10)$. From Figure 2, it is seen that the bipartite consensus of system (3) can be asymptotically reached after a certain amount of time. In other words, position states of agents $1,2,3$ and agents 4,5 converge to the same value, respectively. Meanwhile, velocity states of all agents converge to zero.

When all communication delays are $\tau_{15}=0.6$, $\tau_{21}=0.6, \tau_{32}=0.65, \tau_{43}=0.65, \tau_{52}=0.6$, and $\tau_{54}=0.6$, it takes more time to achieve the same value for position states of agents in Figure 3(a), respectively. From Figure 3(b), it is seen that the time required is increased for the velocity states of all agents reaching zero.

Example 2. Under structural unbalance, a second-order strongly connected multiagent system (3) with signed digraph is considered as shown in Figure 1(b), which contains 5 agents. The corresponding adjacency matrix is denoted as

$$
A_{b}=\left(\begin{array}{ccccc}
0 & 0 & 0 & 0 & 1.2 \\
1.5 & 0 & 0 & 0 & 0 \\
0 & -1 & 0 & 0 & 0 \\
0 & 0 & 1 & 0 & 0 \\
0 & -3 & 0 & -1.3 & 0
\end{array}\right) .
$$

Choose that all communication delays are $\tau_{15}=0.1, \tau_{21}=0.3, \tau_{32}=0.25, \quad \tau_{43}=0.15, \tau_{52}=0.1$, and $\tau_{54}=0.1$. Similarly, using proposed nonlinear control protocol (5), the consensus of the system can be achieved for the same initial conditions and the parameters. From Figure 4 , it is obvious that the position and velocity states of all agents realize the same value gradually through a certain time. 


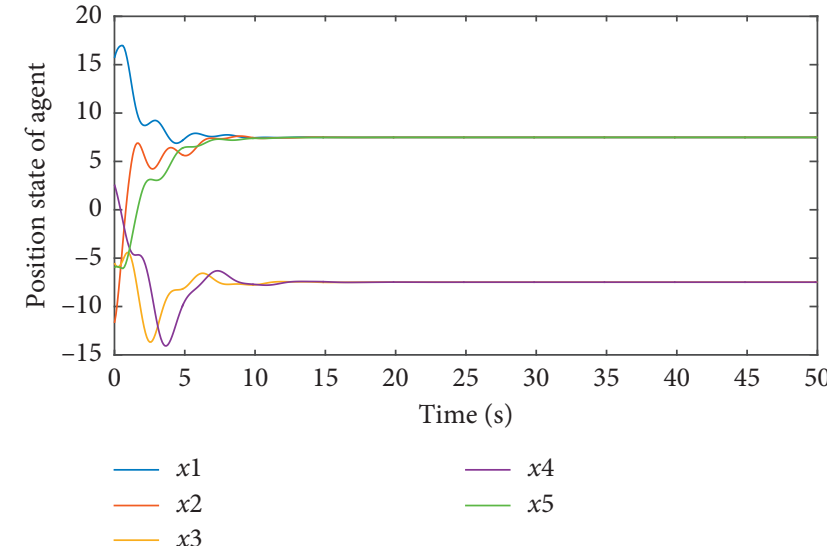

(a)

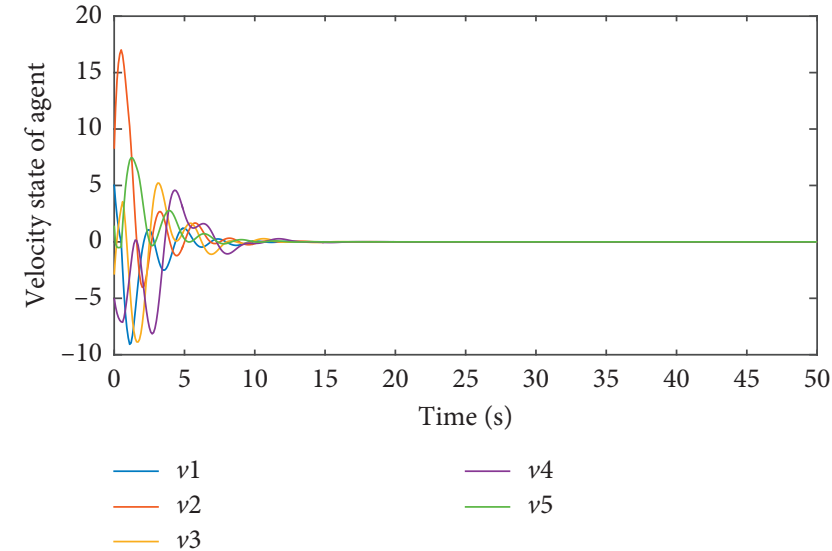

(b)

FIgURE 3: Position and velocity states of agents on the structurally balanced network in Figure 1(a).

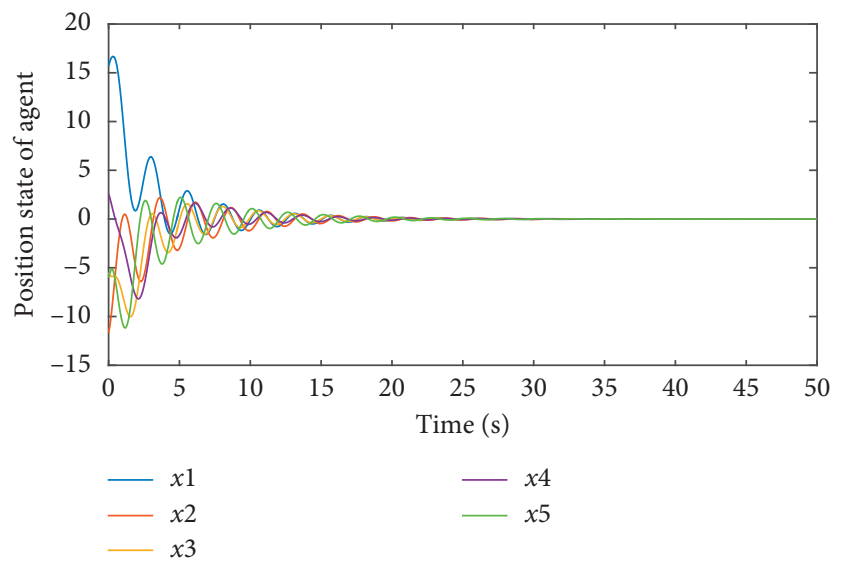

(a)

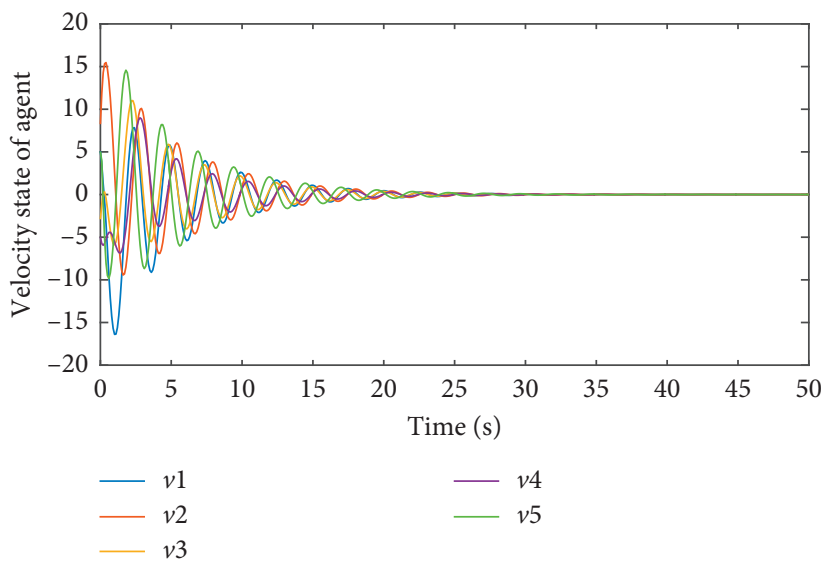

(b)

FIgURe 4: Position and velocity states of agents on structurally unbalanced network in Figure 1(b).

\section{Conclusion}

In this paper, the consensus of second-order MASs with strongly connected signed digraph and communication delays has been studied. The corresponding distributed nonlinear consensus control protocol has been designed for the achievement of second-order MASs with directed signed networks. In addition, using the Lyapunov method, the consensus of second-order MASs with signed digraph and local delays has been certified under structurally balanced and unbalanced networks. Finally, the effectiveness of main results has been further verified via simulation examples.

\section{Data Availability}

The data used to support the findings of this study are included within the article.

\section{Conflicts of Interest}

The authors declare that they have no conflicts of interest.

\section{Acknowledgments}

This work was supported in part by the Natural Science Foundation of Jiangxi Province under Grant 20192BAB217009, the Science and Technology Research Project of Jiangxi Provincial Educational Department under Grant GJJ180349, and the Doctoral Scientific Research Foundation of East China Jiaotong University under Grant 2003418002.

\section{References}

[1] C. W. Reynolds, "Flocks, herds and schools: a distributed behavioral model," ACM SIGGRAPH Computer Graphics, vol. 21, no. 4, pp. 25-34, 1987.

[2] B. Ning, Q.-L. Han, Z. Zuo, J. Jin, and J. Zheng, "Collective behaviors of mobile robots beyond the nearest neighbor rules with switching topology," IEEE Transactions on Cybernetics, vol. 48, no. 5, pp. 1577-1590, 2018.

[3] X. Dong, Y. Hua, Y. Zhou, Z. Ren, and Y. Zhong, "Theory and experiment on formation-containment control of multiple multirotor unmanned aerial vehicle systems," IEEE 
Transactions on Automation Science and Engineering, vol. 16, no. 1, pp. 229-240, 2019.

[4] S. Dhuli, K. Gaurav, and Y. N. Singh, "Convergence analysis for regular wireless consensus networks," IEEE Sensors Journal, vol. 15, no. 8, pp. 4522-4531, 2015.

[5] R. Olfati-Saber and R. M. Murray, "Consensus problems in networks of agents with switching topology and time-delays," IEEE Transactions on Automatic Control, vol. 49, no. 9, pp. 1520-1533, 2004.

[6] W. Yu, W. Ren, W. X. Zheng, G. Chen, and J. Lü, "Distributed control gains design for consensus in multi-agent systems with second-order nonlinear dynamics," Automatica, vol. 49, no. 7, pp. 2107-2115, 2013.

[7] H. Lü, S. Chen, and Q. Lai, "Multi-target consensus circle pursuit for multi-agent systems via a distributed multiflocking method," International Journal of Systems Science, vol. 47, no. 16, pp. 3741-3748, 2016.

[8] Y. Zheng, J. Ma, and L. Wang, "Consensus of hybrid multiagent systems," IEEE Transactions on Neural Networks and Learning Systems, vol. 29, no. 4, pp. 1359-1365, 2018.

[9] H. Pei, S. Chen, Q. Lai, and H. Yan, "Consensus tracking for heterogeneous interdependent group systems," IEEE Transactions on Cybernetics, vol. 50, no. 4, pp. 1752-1760, 2020.

[10] Y. Sun, H. Su, and S. Chen, "Semi-global observer-based nonnegative edge-consensus of linear discrete-time multiagent systems with nonnegative constraint and input saturation," Neurocomputing, vol. 339, pp. 36-44, 2019.

[11] M. E. Valcher and P. Misra, "On the consensus and bipartite consensus in high-order multi-agent dynamical systems with antagonistic interactions," Systems \& Control Letters, vol. 66, pp. 94-103, 2014.

[12] C. Altafini, "Consensus problems on networks with antagonistic interactions," IEEE Transactions on Automatic Control, vol. 58, no. 4, pp. 935-946, 2013.

[13] D. Meng, M. Du, and Y. Jia, "Interval bipartite consensus of networked agents associated with signed digraphs," IEEE Transactions on Automatic Control, vol. 61, no. 12, pp. 3755-3770, 2016.

[14] L. Wang and F. Xiao, "Finite-time consensus problems for networks of dynamic agents," IEEE Transactions on Automatic Control, vol. 55, no. 4, pp. 950-955, 2010.

[15] D. Meng, Y. Jia, and J. Du, "Finite-time consensus for multiagent systems with cooperative and antagonistic interactions," IEEE Transactions on Neural Networks and Learning Systems, vol. 27, no. 4, pp. 762-770, 2016.

[16] H. Wang, W. Yu, G. Wen, and G. Chen, "Finite-time bipartite consensus for multi-agent systems on directed signed networks," IEEE Transactions on Circuits and Systems I: Regular Papers, vol. 65, no. 12, pp. 4336-4348, 2018.

[17] J. Lu, X. Guo, T. Huang, and Z. Wang, "Consensus of signed networked multi-agent systems with nonlinear coupling and communication delays," Applied Mathematics and Computation, vol. 350, pp. 153-162, 2019.

[18] X. Guo, J. Lu, A. Alsaedi, and F. E. Alsaadi, "Bipartite consensus for multi-agent systems with antagonistic interactions and communication delays," Physica A: Statistical Mechanics and Its Applications, vol. 495, pp. 488-497, 2018.

[19] V. M. Popov and R. Georgescu, Hyperstability of Control Systems, Springer-Verlag, Berlin, Germany, 1973.

[20] W. Yu, G. Chen, M. Cao, and W. Ren, "Delay-induced consensus and quasi-consensus in multi-agent dynamical systems," IEEE Transactions on Circuits and Systems I: Regular Papers, vol. 60, no. 10, pp. 2679-2687, 2013.
[21] K. Liu, A. Seuret, Y. Xia, F. Gouaisbaut, and Y. Ariba, "Bessellaguerre inequality and its application to systems with infinite distributed delays," Automatica, vol. 109, Article ID 108562, 2019.

[22] K. Liu, A. Selivanov, and E. Fridman, "Survey on time-delay approach to networked control," Annual Reviews in Control, vol. 48, pp. 57-79, 2019. 